\title{
Abordagem odontológica da cárie de radiação oriunda de efeitos adversos da radioterapia de cabeça e pescoço
}

\author{
Dental Approach to Radiation Carie from Adverse \\ Effects of Head and Neck Radiotherapy
}

\section{RESUMO}

Introdução: Utiliza-se radioterapia para combate ao câncer de cabeça e pescoço. Efeitos adversos agudos e tardios podem ser encontrados em decorrência de intervenção radioterápica em cabeça e pescoço. Os tardios englobam a ocorrência da cárie de radiação, trismo, fibrose cutânea, edema da face e do pescoço, sintomatologia dolorosa e osteorradionecrose, entre outras. Objetivo: O objetivo deste artigo foi evidenciar como ocorre acometimento por cárie de radiação nos pacientes advindo do emprego de tratamento radioterápico antineoplásico na região de cabeça e pescoço. Metodologia: Realizou-se revisão bibliográfica com levantamento nas bases de dados: LILACS, Google Acadêmico de estudos e artigos que versavam acerca do acometimento da cárie de radiação advinda de efeito adverso da radioterapia. Resultados: A cárie de radiação advém de alterações desfavoráveis do $\mathrm{pH}$ e da elevação do quantitativo de bactérias cariogênicas, que é oriundo das sessões de radioterapia, levando ao aumento de biofilme dentário na porção cervical dos dentes, podendo ocasionar locais com dentina exposta. O motivo principal para que cáries de radiação ocorram provém de modificações quantitativas e qualitativas da saliva. Convive-se com 3 tipos de cárie de radiação: tipo 1, 2 e 3 . O tratamento da cárie de radiação envolve realização de bochechos com soluções fluoretadas e administração tópica de flúor gel neutro. O tratamento curativo emprega cimento de ionômero de vidro modificado por resina. Conclusão: O cirurgião dentista deve possuir embasamento prévio e conscientizar-se acerca da possibilidade do acometimento pela cárie de radiação em pacientes que se submeteram a tratamento oncológico radioterápico.

Palavras-chave: Neoplasias de Cabeça e Pescoço. Radioterapia. Doses de Radiação. Saliva.

\section{ABSTRACT}

Introduction: Radiotherapy is used to fight head and neck cancer. Acute and late adverse effects can be found as a result of radiotherapy intervention in the head and neck. The late ones include the occurrence of radiation caries, trismus, cutaneous fibrosis, edema of the face and neck, painful symptoms and osteoradionecrosis, among others. Objective: The objective of this article was to show how radiation caries affects patients in the treatment of head and neck cancer. Methodology: A bibliographic review was carried out with a survey in the databases: LILACS, Google Scholar of studies and articles dealing with the involvement of radiation caries resulting from the adverse effect of radiotherapy. Results: Radiation decay results from unfavorable changes in $\mathrm{pH}$ and an increase in the amount of cariogenic bacteria, which comes from radiotherapy sessions, leading to an increase in dental biofilm in the cervical portion of the teeth, which can cause exposed dentin. The main reason for radiation caries to occur comes from quantitative and qualitative changes in saliva. There are 3 types of radiation caries: types 1,2 and 3. The treatment of radiation caries involves mouthwashing with fluoridated solutions and topical administration of neutral fluoride gel. The curative treatment uses resin-modified glass ionomer cement. Conclusion: The dental surgeon must have previous knowledge and be aware of the possibility of being affected by radiation caries in patients who have undergone radiotherapy oncological treatment.

Keywords: Head and Neck Neoplasms. Radiotherapy. Radiation Dosage. Saliva.

\section{Sérgio Spezzia ${ }^{1}$}

${ }^{1}$ Cirurgião Dentista. Especialista em Saúde da Mulher no Climatério pela Faculdade de Saúde Pública da Universidade de São Paulo. Especialista em Gestão Pública pela Universidade Tecnológica Federal do Paraná. Especialista em Gestão em Saúde pela Universidade Federal de São Paulo. Especialista em Adolescência para Equipe Multidisciplinar e Mestre em Ciências pela Escola Paulista de Medicina - Universidade Federal de São Paulo.

Endereço para Correspondência: Sérgio Spezzia

Rua Silva Bueno, 1001, Ipiranga, CEP 04208-

050, São Paulo, SP

sergio.spezzia@unifesp.br

Submetido em: 30-8-2020

Aceito em: 7-5-2021 


\section{INTRODUÇÃO}

A terapia para tratamento do câncer na região de cabeça e pescoço pode ocorrer com utilização somente de cirurgia ou com o emprego da cirurgia coadjuvada a quimioterapia e a radioterapia ${ }^{1-3}$. Sabe-se que a quimioterapia e a radioterapia objetivam deter e destruir o tumor, entretanto, esses tratamentos antineoplásicos podem ocasionar também efeitos indesejáveis aos tecidos sadios circunvizinhos ${ }^{4}$. A escolha do tipo de tratamento a ser utilizado levará em consideração o estadiamento, a localização, as características histológicas presentes, o estado de saúde apresentado pelos indivíduos com esse tipo de câncer e a possibilidade de contar-se com a cooperabilidade dos pacientes durante o tratamento ${ }^{5,6}$.

Muito comumente utiliza-se da radioterapia para combate ao câncer de cabeça e pescoço. O local do tumor será submetido a dose de radiação aferida em gray e planejada pelo médico radioterapeuta. A intervenção planejada pelo radioterapeuta deve procurar não afligir tecidos saudáveis circunvizinhos ao tumor, uma vez que na hipótese dessa ocorrência instalam-se efeitos deletérios denominados adversos ou colaterais. A conduta aplicada para enfrentamento do câncer de cabeça e pescoço pode optar pelo uso de doses elevadas. Em decorrência convive-se com efeitos adversos, que podem manifestar-se no transcorrer da terapia ou em período de semanas subsequentes a ela, designado por manifestações de fase aguda. Outra forma de manifestação adversa é evidenciada posteriormente e depois de período que engloba meses ou anos de findada a radioterapia ${ }^{7-13}$.

A abordagem odontológica realizada em pacientes que receberão radioterapia deve ocorrer previamente ao início da terapia para adequação do meio bucal, visando evitar-se maiores complicações posteriormente ${ }^{14-19}$. Efeitos agudos comumente encontrados em decorrência de intervenção radioterápica em cabeça e pescoço envolvem: mucosite oral, disfagia, odinofagia e possibilidade da instalação de infecções oportunistas, entre outros. Outros efeitos são designados de tardios e englobam ocorrência da cárie de radiação, trismo, fibrose cutânea, edema da face e do pescoço, sintomatologia dolorosa e osteorradionecrose, entre outras ${ }^{7,20-24}$.

O cirurgião dentista deve proceder a avaliação e ao acompanhamento dos pacientes submetidos a tratamento radioterápico antineoplásico na região de cabeça e pescoço, tanto em período anterior ao início do tratamento, como no transcorrer e depois de findado o mesmo $^{23,25-29}$.

O objetivo deste artigo foi evidenciar como ocorre o acometimento por cárie de radiação nos pacientes advindo do emprego de tratamento radioterápico antineoplásico na região de cabeça e pescoço.

\section{METODOLOGIA}

Realizou-se revisão bibliográfica com levantamento nas bases de dados: Literatura Latino-americana e do Caribe em Ciências da Saúde (LILACS), Google Acadêmico de estudos e artigos que versavam acerca do acometimento da cárie de radiação advinda de efeito adverso da radioterapia. No Google Acadêmico a expressão de busca empregada foi: cárie de radiação and radioterapia and efeitos adversos and câncer and tratamento 
antineoplásico and odontologia and oncologia and tratamento odontológico and 2020 and 2019 and 2018 and 2017 and 2016 e obteve-se aproximadamente 52 resultados. No LILACS utilizou-se a expressão de busca: cárie de radiação and radioterapia e encontrou-se 48 resultados.

Incluiu-se artigos publicados nos idiomas inglês e português em periódicos internacionais e nacionais que tratavam do acometimento de cárie de radiação oriunda de intervenção radioterápica.

Excluiu-se artigos que não possuíam conteúdo concernente com o pesquisado ou que tratavam de outras temáticas.

Apontamentos de livros, trabalhos, monografias, dissertações e teses que abordavam essa mesma temática também foram considerados válidos.

\section{REVISÃO DE LITERATURA}

A radioterapia produz efeitos colaterais que variam em conformidade com a quantia de radiação aplicada; a faixa etária dos doentes em tratamento; a forma de aplicação da dose se fracionada ou não e às características da região irradiada, entre outros fatores influenciadores. Pode-se designar esses efeitos em efeitos agudos, que se manifestam logo nas semanas subsequentes ao começo das intervenções radioterápicas e em efeitos tardios, que são verificados somente passados meses ou anos de findada a radioterapia, entre esses efeitos tardios pode ocorrer o acometimento pela cárie de radiação ${ }^{30-33}$.

A nível odontológico deve-se primar por uma abordagem preventiva dos pacientes anteriormente ao começo das sessões de radioterapia, firmando-se o diagnóstico dos problemas odontológicos que necessitam ser tratados e procedendo-se ao feitio preferencialmente de todos os procedimentos odontológicos em fase preliminar ${ }^{17,22,23,34,35}$. Procedimentos odontológicos mais urgentes que precisam ser efetuados para evitar-se ocorrência de complicações futuras oriundas do tratamento antineoplásico devem serem realizados. Priorizam-se para tratamento situações clínicas que ocasionem processos dentários infecciosos; deve-se proceder ao tratamento dos processos inflamatórios presentes; realizam-se os tratamentos endodônticos que forem necessários; cáries dentárias extensas devem ser tratadas; deve-se eliminar próteses dentárias com má adaptação e que causam trauma a mucosa bucal; prima-se por evitar qualquer evidência provável da instalação de infecções oportunistas, dentre outros cuidados ${ }^{17,22,35}$. No mais, os pacientes devem receber orientações acerca dos cuidados essenciais de higiene bucal, visando que os mesmos possam ter meios de aperfeiçoar a forma como realizam o seu autocuidado voltado para sua higienização oral. A higienização bucal deve ser realizada suficientemente por parte dos pacientes seja antes, durante ou depois das intervenções de radioterapia e para tal é necessário realizar embasamento dos pacientes previamente por intermédio de instruções por parte do cirurgião dentista sobre como fazer para proceder ao feitio de uma higienização bucal de forma correta e vigorosa utilizando de técnicas de escovação dentária e do emprego do fio e da fita dental adequadamente ${ }^{34,35,36-38}$.

Deve-se ainda enfatizar que nessas situações o emprego de flúor e de clorexidina mostra-se satisfatório como proteção para evitar ocorrência da cárie de radiação. Emprega-se preventivamente flúor neutro que deve ser aplicado topicamente sobre os dentes e 
recomenda-se o feitio de bochechos com solução fluoretada ${ }^{23,39-41}$. No mais, deve-se realizar acompanhamento periódico dos pacientes em consultas odontológicas intervaladas, visando minimizar a possibilidade de instalação da cárie de radiação, entretanto, mesmo assim existem ainda chances para que as cáries de radiação ocorram, mesmo adotando todos esses cuidados $8,14,17,18,28,35,37,39$.

A cárie de radiação consta de complicação tardia das aplicações de radioterapia para combate ao câncer de cabeça e pescoço e advém de alterações desfavoráveis do pH e da elevação do quantitativo de bactérias, que é oriundo ou provocado pelas sessões de radioterapia, levando ao aumento de biofilme dentário na porção cervical dos dentes $3,23,28,40,42-45$. O motivo principal para que essas cáries instalem-se provém de modificações quantitativas e qualitativas da saliva. Nessa situação a saliva deixa de exercer o seu papel de tampão e de controle do $\mathrm{pH}$ bucal, ocasionando possivelmente modificações nos constituintes inorgânicos e orgânicos dos elementos dentais, podendo levar a descalcificação $22,30,42,44,46,47$. A evolução desse tipo de cárie pode originar o acometimento por perdas dentárias ${ }^{3,40,42,43}$. O aspecto clínico da cárie de radiação é similar ao comumente presente nas cáries dentárias. Ela aflige frequentemente as superfícies lisas e cervicais localizadas em molares, incisivos e pré-molares. As mesmas afligem comumente as porções cervicais dos dentes $3,22,23,28,44,45$. Os indivíduos podem sofrer vários problemas de ordem odontológica, advindo da presença da cárie de radiação, devido ao fato a mesma deve receber o devido tratamento já em sua fase de início para que esses problemas não se agravem. A sua evolução é acelerada e pode afligir a polpa dentária, possivelmente podendo acarretar necessidade de proceder-se ao tratamento endodôntico. Outros problemas apresentados envolvem a possibilidade dessa cárie poder causar prejuízo estético e fornecer situação de vulnerabilidade para a instalação de fraturas dentárias, entre outras ocorrências. As consequências dessa cárie podem levar a ocorrência de perdas dentárias $3,24,42,48$.

Convive-se com 3 tipos de cárie de radiação: tipo 1, tipo 2 e tipo 3 . O tipo 1 consta do mais frequente e aflige a porção cervical dos dentes indo até a junção amelo-cementária. Já no tipo 2, cáries são evidenciadas em todas as partes nas superfícies dos dentes e averiguam-se locais com presença de desmineralização. Superfícies incisais e oclusais são acometidas. O tipo 3 pouco comumente ocorre, nele verificam-se desgastes em oclusal dos dentes e modificações de cor em dentina, que assume coloração marrom escura ${ }^{23}$.

O tratamento da cárie de radiação envolve a realização de bochechos com soluções fluoretadas e a administração tópica de flúor em gel neutro. O tratamento odontológico curativo emprega restaurações com cimento de ionômero de vidro modificado por resina, devido às propriedades satisfatórias desse material odontológico, que permitem liberação e reincorporação de flúor $23,28,39,40,49-52$.

\section{DISCUSS ÃO}

A terapia antineoplásica realizada por intermédio de sessões de radioterapia e quimioterapia requer atenção de equipe multidisciplinar, equipe na qual deve estar inserido o cirurgião dentista, uma vez que o mesmo terá papel fundamental para realização de controle e avaliação da saúde bucal dos pacientes ${ }^{39,41,49,53-56}$. 
A abordagem odontológica preliminar dos pacientes deve levantar a história médica dos mesmos, procurando informações mais detalhadas acerca do tumor presente e sobre o tratamento oncológico que será realizado, bem como sobre qual será a medicação prescrita, a dose de radiação empregada e o período de duração das sessões de radioterapia, entre outros dados. Baseado nesse levantamento deve-se realizar um planejamento visando adequação do meio bucal dos pacientes cancerosos, no intuito de minimizar eventuais complicações orais indesejáveis oriundas do tratamento antineoplásico ${ }^{8,28,23,30,31,37,49,55-59}$.

Complicações originadas pela radioterapia de cabeça e pescoço, dentre as quais a cárie de radiação afetam negativamente a qualidade de vida dos pacientes. Os cirurgiões dentistas devem procurar embasar-se acerca de todos possíveis efeitos adversos capazes de serem gerados por esse tipo de radioterapia para que possam elaborar um planejamento odontológico dotado de abordagens e procedimentos tanto com cunho preventivo como também com enfoque curativo. Efeitos adversos ocasionados pelo emprego da radioterapia em boca podem afligir sobremaneira a qualidade de vida dos pacientes, podendo haver manifestações de sintomatologia dolorosa, bem como de ocorrência de perdas dentárias $^{8,14,17,30,32,60,61}$.

Estudo realizado por Palmier et al., $(2017)^{62}$, procedeu a classificação da cárie de radiação em conformidade com o Sistema Internacional de Detecção e Avaliação de Cárie (ICDAS) e em conformidade com o Índice Dental Pós-radiação (PRDI), almejando com essas medidas aferir e averiguar a maneira como esse tipo de cárie é capaz de evoluir. Os autores examinaram 883 elementos dentais de indivíduos que tinham recebido tratamento radioterápico na região de cabeça e pescoço com dose de radiação administrada oscilando entre 60 e 70 gray. Depois de findada a radioterapia, desses 883 dentes, 814 tiveram diagnóstico clínico de cárie de radiação. Realizadas as avaliações baseadas no ICDAS e no PRDI, os pesquisadores puderam verificar que a cárie de radiação demonstra ser uma doença dotada de certa agressividade.

Existem evidências científicas que afirmam que uma vez findada a radioterapia, torna-se vigente a provável ocorrência da cárie de radiação posteriormente por todo o período no decorrer de suas vidas nos indivíduos que receberam esse tipo de tratamento ${ }^{63}$.

Em conformidade com Beech et al., $(2014)^{49}$, não existe um material odontológico restaurador para tratamento da cárie de radiação que se mostre ideal e que preencha todos os requisitos almejados, principalmente em termos de estética e durabilidade e no que tange ao impedimento da instalação subsequente de cáries secundárias.

O cimento de ionômero de vidro possui propriedade anticariogênica de liberação de flúor, o que pode auxiliar na minimização das chances de ocorrer cárie secundária, em contrapartida esse cimento constitui um material que não possui propriedades satisfatórias de resistência ${ }^{50}$.

\section{CONCLUSÕES}

Ao proceder ao tratamento radioterápico de neoplasias malignas na região de cabeça e pescoço, concomitantemente pode-se conviver com efeitos indesejáveis em âmbito bucal, estes que chegam a comprometer por vezes a qualidade de vida dos indivíduos. 
O cirurgião dentista deve possuir embasamento prévio e conscientizar-se acerca da possibilidade do acometimento pela cárie de radiação em pacientes que se submeteram a tratamento oncológico radioterápico.

Uma anamnese detalhada previamente a qualquer abordagem odontológica deve averiguar a história médica dos pacientes, verificando quais doenças sistêmicas foram tratadas ou encontram-se em tratamento, deve-se avaliar dentre estas a possibilidade do acometimento por câncer de cabeça e pescoço e o subsequente tratamento radioterápico, circunstância na qual deve-se empreender atenção odontológica redobrada.

Um planejamento médico bem delineado para a execução da radioterapia na região de cabeça e pescoço pode minimizar ou evitar os riscos para a instalação da cárie de radiação.

\section{REFERÊNCIAS}

1. Villar CMC, Martins IM. Princípios de cirurgia oncológica. In: Vieira SC, Lustosa AML, Barbosa CNB, Teixeira JMR, Brito LXE, Soares LFM, et al. Oncologia Básica. 1ª. ed. Teresina: Fundação Quixote, 2012.

2. Brasil. Ministério da Saúde/ Secretaria de Atenção à Saúde/ Departamento de Regulação, Avaliação e Controle/ Coordenação Geral de Sistemas de Informação. Manual de Bases Técnicas da Oncologia - SIA/SUS - Sistema de Informações Ambulatoriais, 2013. 116 ps.

3. Dobros K, Hajto-BryK J, Wroblewska M, Zarzecka J. Radiation-induced caries as the late effect of radiation therapy in the head and neck region. Contemp Oncol (Pozn), 2016; 20(4):287-90.

4. Volpato LER, Silva TC, Oliveira TM, Sakai VT, Machado MAAM. Mucosite bucal rádio e quimioinduzida. Rev Bras Otorrinolaringol, 2007; 73(4):562-8.

5. Lobo ALG, Martins GB. Consequências da Radioterapia na Região de Cabeça e Pescoço: uma revisão da literatura. Rev Portug Estomatol Med Dent Cir Maxilofac, 2009; 50(4):251-5.

6. Sassi LM, Machado RA. Protocolo pré-radioterapia de cabeça e pescoço. Rev Bras Cir Cabeça e Pescoço, 2009; 38(3):208-10.

7. Spetch L. Oral complications in the head and neck irradiated patient. Introduction and scope of the problem. Supp Care Dent, 2002; 10:36-9.

8. Jham BC, Freire ARS. Complicações bucais da radioterapia em cabeça e pescoço. Rev Bras Otorrinolaringol, 2006; 5(72):704-08.

9. Guimarães JRQ. Manual de oncologia. 2ª ed. São Paulo: BBS, 2006.

10. Waldron J, O'Sullivan B. Princípios da radioterapia oncológica. In: União Internacional contra o Câncer. Manual de oncologia da clínica da UICC. 8a. ed. São Paulo: UICC; 2008.

11. Tauhata L, Salati IP, di Prinzio R. Radioproteção e dosimetria: fundamentos. 9ª rev. Rio de Janeiro: IRD/CNEN; 2013.

12. Spezzia S. O uso do bolus na radioterapia. Rev Fac Ciênc Méd Sorocaba, 2016; 18(4):183-6.

13. Brennan PA, Bradley KL, Brands M. Intensity-modulated radiotherapy in head and neck cancer - an update for oral and maxillofacial surgeons. Br J Oral Maxillofac Surg, 2017; 55(8):770-4.

14. Epstein JB, Robertson M, Emerton S, Phillips N, Stevenson-Moore P. Quality of life and oral function in patients treated with radiation therapy for head and neck cancer. Head Neck, 2001; 23(5):389-98.

15. Bonan PR, Lopes MA, Pires FR, Almeida OP. Dental management of low socioeconomic level patients before radiotherapy of the head and neck with special emphasis on the prevention of osteoradionecrosis. Braz Dent J, 2006; 17(4):336-42

16. Caccelli EMN, Pereira MLM, Rapoport A. Avaliação da mucosite e xerostomia como complicações do tratamento de radioterapia no câncer de boca e orofaringe. Rev Bras Cir Cab Pesc, 2009; 38(2):80-3.

17. Matsuzaki H, Tanaka-Matsuzaki K, Miyazaki F, Aoyama H, Ihara H, Katayama N et al. The role of dentistry other than oral care in patients undergoing radiotherapy for head and neck cancer. Jap Dent Sci Review, 2017; 53(2):46-52.

18. Mercadante V, Al Hamad A, Lodi G, Porter S, Fedele S. Interventions for the management of radiotherapy-induced xerostomia and hyposalivation: a systematic review and meta-analysis. Oral Oncology, 2017; 66:64-74.

19. Villa A, Akintoye SO. Dental Management of Patients Who Have Undergone Oral Cancer Therapy. Dent Clin North Am, 2018; 62(1):131-42.

20. Geraldes AM. Ocorrência de Porphyromonas gingivalis na microbiota bucal de pacientes submetidos à radioterapia para tratamento de lesões malignas de cabeça e pescoço. [Trabalho de conclusão de curso]. Araçatuba: Faculdade de Odontologia de Araçatuba - Universidade Estadual Paulista Julio de Mesquita Filho - UNESP, 2010. 
21. Marta GN, Hanna SA, Silva JLF, Carvalho HA. Câncer de cabeça e pescoço e radioterapia: breve contextualização. Diagn Tratamento, 2011; 16(3):134-6.

22. Rolim AEH, Costa LJ, Ramalho LMP. Repercussões da radioterapia na região orofacial e seu tratamento. Radiol Bras, 2011; 44(6):388-95.

23. Gupta N, Pal M, Rawat S, Grewal MS, Garg H, Chauan D et al. Radiation-induced dental caries, prevention and treatment - a systematic review. Natl J MaxillofacSurg, 2015; 6(2):160-6.

24. Morais-Faria K, Neves-Silva R, Lopes MA, Ribeiro ACP, de Castro Jr. G, Vasconcelos KGMC et al. The wolf in sheep's clothing: microtomographic aspects of clinically incipient radiation-related caries. Med Oral Patol Oral Cir Bucal, 2016; 21(3):e299-e304.

25. Cardoso MFA, Novikoff S, Tresso A, Segreto RA, Cervantes O. Prevenção e controle das sequelas bucais em pacientes irradiados por tumores de cabeça e pescoço. Radiol Bras, 2005; 38(2):107-15.

26. Joshi VK. Dental treatment planning and management for the mouth cancer patient. Oral Oncol, 2010; 46:475-9

27. Walsh LJ. Clinical assesment and management on the oral environment in the oncology patient. Aust Dent J, 2010; 55:66-77.

28. Tolentino ES, Centurion BS, Ferreira LHC, de Souza AP, Damante JH, Rubira-Bullen IRF. Oral adverse effects of head and neck radiotherapy: literature review and suggestion of a clinical oral care guideline for irradiated patients. J Appl Oral Sci, 2011; 19(5):448-54.

29. Vieira DL, Leite AF, Melo NS, Figueiredo PTS. Tratamento odontológico em pacientes oncológicos. Oral Sci, 2012; $4(2): 37-42$.

30. Salazar M, Victorino FR, Paranhos LR, Ricci ID, Gaeti WP, Caçador NP. Efeitos e tratamento da radioterapia de cabeça e pescoço de interesse ao cirurgião dentista: revisão da literatura. Rev Odonto, 2008; 16(31):62-8.

31. Santos MG, Silva LCF, Lins CA, Passos DD, Oliveira Neto JN, Santos TS. Fatores de risco em radioterapia de cabeça e pescoço. RGO, 2010; 58(2):191-6.

32. Bragante KC, Nascimento DM, Motta NW. Avaliação dos efeitos agudos da radioterapia sobre os movimentos mandibulares de pacientes com câncer de cabeça e pescoço. Rev Bras Fisioter, 2011. Disponível em: https://www. scielo.br/pdf/rbfis/2012nahead/pt_aop014_12.pdf Acessado em 21 de agosto de 2020.

33. Bueno AC, Magalhães CS, Cláudia; Moreira NA. Associações entre Fatores de Risco e Complicações Bucais em Pacientes com Câncer de Cabeça e Pescoço Tratados com Radioterapia Associada ou Não à Quimioterapia. Pesq Bras Odontoped Clín Int, 2012; 12(2):187-93.

34. Wright WE, Haller JM, Harlow SA, Pizzo PA. An oral disease prevention program for patients receiving radiation and chemotherapy. J Am Dent Assoc, 1985; 110:43-7.

35. Faloni APS, Lorenzon AP, Margonar R. Importância dos procedimentos periodontais prévios à radioterapia em região de cabeça e pescoço. Rev Int Periodontia Clin, 2005; 2:93-9.

36. Dib LL, Gonçalves RCC, Kowalski LP, Salvajoli JV. Abordagem multidisciplinar das complicações orais da radioterapia. Rev Assoc Paul Cir Dent, 2000; 54:391-6.

37. Albuquerque RA, Morais VLL, Sobral APV. Protocolo de atendimento odontológico a pacientes oncológicos pediátricos - revisão da literatura. Rev Odontol UNESP, 2007; 36:275-80.

38. de Souza RC. Interfaces entre a Radioterapia e a Odontologia. Especialização em Radiologia e Imaginologia. [Monografia]. Porto Alegre: Faculdade de Odontologia da Universidade Federal do Rio Grande do Sul, 2011.

39. Andrews N, Griffths C. Dental complications of head and neck radiotherapy: Part 1. Austr Dent J, 2001; 46(2):88-94.

40. Antonio AMMP, Maia FAZ, Dias RB. Reações adversas da radioterapia: cuidados pré, trans e pós operatório. Rev Odonto. 2001; 9:12-9.

41. Rubira CM, Devides NJ, Ubeda LT, Bortolucci AG Jr, Lauris JR, Rubira-Bullen IR, et al. Avaliação de algumas sequelas pós-radioterapia oral em pacientes tratados de tumores de cabeça e pescoço. Braz Oral Res, 2007; 21(3):272-7.

42. Sonis ST, Fazio RC, Fang L. Princípios e prática de medicina oral. 2a . ed. Rio de Janeiro: Guanabara Koogan, 1996. p. 335-49.

43. Santos ACM, Noguti F, Mendes FCA, Alborghetti GP, Pereira MB. Complicações Bucais no Tratamento Radioterápico. 2002. Disponível em: http://www.dracarolinafernandes.com.br/dicas/oncologialaserterapia/ Complica\%E7\%F5es\%20Bucais\%20no\%20Tratamento\%20Radioter\%E1pico.pdf Acessado em 21 de agosto de 2020.

44. Osterne, RLV. et al. Saúde bucal em portadores de neoplasias malignas: estudo clínico-epidemiológico e análise das necessidades odontológicas de 421 pacientes. Rev Bras Cancerol, 2008; 54(3):221-6.

45. Neville BW. Patologia oral e maxilofacial. 3a. ed. Rio de Janeiro: Guanabara Koogan, 2009. p. 287-330.

46. Epstein JB, Chin EA, Jacobson JJ, Rishiraj B, Le N. The relationships among fluoride, cariogenic oral flora, and salivary flow during radiation therapy. Oral Surg Oral Med Oral Pathol, 1998; 86:286-92.

47. Silverman S Jr. Oral cancer. Complications of therapy. Oral Surg Oral Med Oral Pathol Oral Radiol Endond, 1999; 88:122-6. 
48. Caielli C, Martha PM. Sequelas orais da radioterapia: atuação da odontologia na prevenção e tratamento. Rev Bras Cancerol, 1995; 4(41):231-41.

49. Beech N, Robinson S, Porceddu S, Batstone M. Dental management of patients irradiated for head and neck cancer. Austr Dent J, 2014; 59(1):20-8.

50. Spezzia S. Cimento de ionômero de vidro: revisão de literatura. J Oral Inv, 2017; 6(2): 74-88.

51. Spezzia S. Resinas compostas nanoparticuladas. Rev Eletr Odontol Clín Integr UNIRP, 2020; 4(1):96-103.

52. Spezzia S. Sistemas Adesivos. Int J Scien Dent, 2020; 2(54):55-64.

53. Lima AAS, Figueiredo MAZ, Loureiro MS, Duarte R. Radioterapia de neoplasias malignas na região de cabeça e pescoço - o que o cirurgião dentista precisa saber. Rev Odontociênc, 2001; 16:156-63.

54. Ramos FMM, Terra ER, Brayner SC, Haiter Neto F. O papel do cirurgião-dentista na radioterapia de cabeça e pescoço. Odontol Clín Científ, 2005; 4(1):89-94.

55. Spezzia S. Mucosite Oral. J Oral Inv, 2015; 4(1):14-8.

56. Spezzia S. Mucosite Oral em Pacientes Cancerosos Submetidos a Tratamento Quimioterápico. Rev Cienc Odontol, 2020; 4(1):36-40.

57. Andrade CR, Lopes SMP, Coletta RD, Vargas PA, Lopes MA. Tratamento endodôntico em pacientes submetidos à radioterapia na região de cabeça e pescoço. Rev APCD, 2003; 57:43-6.

58. Almeida LHRB, Pereira YBAS, Oliveira TA. Radioterapia: percepção de mulheres com câncer cérvico-uterino. Rev Bras Enf, 2008; 61(4):482-7.

59. Paiva MDEB, de Biase RCCG, Moraes JJC, Ângelo AR, Honorato MCTM. Complicações orais decorrentes da terapia antineoplásica. Arq Odontol, 2016; 46(1):48-55.

60. Sawada NO, Dias AM, Zago MMF. O efeito radioterapia sobre a qualidade de vida dos pacientes com câncer de cabeça e pescoço. Rev Bras Cancerol, 2006; 52(4):323-9.

61. Brennan MT, Woo S-B, Lockhart PB. Dental treatment planning and management in the patient who has cancer. Dent Clin N Am, 2008; 52:19-37.

62. Palmier NR. Aplicabilidade do ICDAS e do Índice Dental Pós-radioterapia na Avaliação de Cárie Relacionada à Radiação. [Dissertação]. Piracicaba: Faculdade de Odontologia de Piracicaba - Universidade Estadual de Campinas, 2017.

63. Kielbassa AM, Hinkelbein W, Hellwig E, Meyer-Lucke H. Radiation - related damage to dentition. Lancet Oncol, 2006; 7(4):326-35. 